\title{
Phenylethanol glycosides from Herba Cistanche improve the hypoxic tumor microenvironment and enhance the effects of oxaliplatin via the HIF-1 $\alpha$ signaling pathway
}

\author{
LIMEI WEN $^{1,2^{*}}$, JUNPING HU $^{1 *}$, JIAWEI ZHANG $^{1}$ and JIANHUA YANG ${ }^{1,2}$ \\ ${ }^{1}$ College of Pharmacy, Xinjiang Medical University, Urumqi, Xinjiang 830054; ${ }^{2}$ Department of Pharmacy, \\ The First Affiliated Hospital, Xinjiang Medical University, Urumqi, Xinjiang 830011, P.R. China
}

Received July 29, 2020; Accepted March 9, 2021

DOI: $10.3892 / \mathrm{mmr} .2021 .12156$

\begin{abstract}
Liver cancer is one of the most common types of malignant tumor, and is characterized by high malignancy, rapid progression, high morbidity and mortality. Oxaliplatin (OXA) has been reported to have marked efficiency against advanced liver cancer with tolerable toxicity. In solid tumors, the hypoxic microenvironment promotes epithelial-mesenchymal transition (EMT), which can also induce drug resistance of liver cancer to platinum drugs. Herba Cistanche (Cistanche tubulosa) has been frequently used in traditional Chinese medicine and the phenylethanol glycosides from Herba Cistanche (CPhGs) are the major active components. The present study aimed to investigate the effects of $\mathrm{CPhGs}$ on viability, apoptosis, migration and invasion of liver cancer cells. HepG2 liver cancer cells were divided into the control, DMSO, $\mathrm{CoCl}_{2}$, OXA, OXA $+\mathrm{CoCl}_{2}$ and $\mathrm{CPhGs}+\mathrm{OXA}+\mathrm{CoCl}_{2}$ groups. Subsequently, reverse transcription-quantitative PCR and western blot analysis were performed to determine the expression levels of hypoxia-inducible factor $1 \alpha(\mathrm{HIF}-1 \alpha)$, lysyl oxidase-like 2 (LOXL2) and EMT-related genes and proteins (i.e., E-cadherin and Twist), in order to investigate the effects of $\mathrm{CPhGs}$ on liver cancer. The results demonstrated that $\mathrm{CPhGs}$ could enhance the effects of OXA on liver cancer, and inhibit the migration, invasion and apoptotic rate of liver cancer cells. Additionally, $\mathrm{CPhGs}$ treatment effectively induced downregulation of HIF-1 $\alpha$, LOXL2 and Twist, and upregulation of E-cadherin. The present findings indicated that $\mathrm{CPhG}$ triggered a significant increase in sensitivity to OXA and suppression of hypoxia-induced EMT in liver
\end{abstract}

Correspondence to: Dr Jianhua Yang, Department of Pharmacy, The First Affiliated Hospital, Xinjiang Medical University, 137 Liyushan South Road, Urumqi, Xinjiang 830011, P.R. China E-mail: 609571241@qq.com

*Contributed equally

Key words: liver cancer, hypoxia, HIF-1 $\alpha$ signaling pathway, epithelial-mesenchymal transition, phenylethanol glycosides from Herba Cistanche cancer by inhibiting the HIF-1 $\alpha$ signaling pathway. Therefore, $\mathrm{CPhGs}$ may be considered an effective platinum drug sensitizer, which could improve chemotherapeutic efficacy in patients with liver cancer.

\section{Introduction}

Liver cancer is a malignant tumor that usually involves the digestive system, and consists of primary and secondary types. Primary liver cancer is divided into hepatocellular carcinoma and intrahepatic cholangiocarcinoma $(1,2)$. Notably, liver cancer is associated with a high incidence and mortality rate worldwide (3). According to the World Health Organization, it is predicted that there will be $>1,000,000$ liver cancer-related deaths by 2030, and of the newly confirmed cases, those in mainland China will account for $46.6 \%$.

Oxaliplatin (OXA) has been reported to exert inhibitory effects on the growth of liver cancer with tolerable toxicity in clinical settings. Nevertheless, the overall efficiency of platinum-based drug therapy is hampered by tumor cell resistance (4). It is well acknowledged that liver cancer exhibits lower sensitivity to chemotherapy compared with other types of cancer. The multidrug resistance of liver cancer has contributed to its resistance to numerous therapeutic agents (5). In a previous study, Xie and Zhong (6) reported that HepG2 cells exhibited poor sensitivity to adriamycin, 5-fluorouracil and cisplatin under hypoxic conditions. Despite the fact that platinum-based chemotherapy agents are the major treatment options for cancer, resistance to these drugs does exist among patients. Furthermore, the prognosis of patients with liver cancer remains poor $(7,8)$. To date, extensive efforts have been made to investigate drug resistance and to improve drug sensitivity in patients with liver cancer.

Under hypoxic conditions, revascularization occurs in cancer cells, which can lead to epithelial-mesenchymal transition (EMT) and vascular mimicry (VM). EMT and VM may subsequently promote invasion and distant metastasis. Moreover, EMT has been speculated to serve a role as the driving force for cancer progression (9). In addition, hypoxia-inducible factors (HIFs) are involved in neovessel formation, energy metabolism, cellular proliferation, invasion and metastasis (10). 
Lysyl oxidase (LOX)-like 2 (LOXL2) protein is a member of the LOX family, and is closely related to the covalent cross-linking of collagen and elastin, which can result in fibrosis and is crucial for the integrity of the extracellular matrix (11). In a previous study, LOXL2 was considered to be closely related to the metastasis of cancer cells (12). Furthermore, LOXL2 has been shown to modulate the pathogenesis and progression of numerous types of malignant cancer though extra- and intra-cellular pathways, which were important indices for the evaluation of poor prognosis $(13,14)$.

Herba Cistanche is a tonic herb commonly distributed in desert regions, which has been frequently used in traditional Chinese medicine $(15,16)$. Cistanche tubulosa (C. tubulosa) is a natural herbal medicine commonly planted in Xinjiang Autonomous Region. The phenylethanol glycosides from Herba Cistanche (CPhGs) serve as one of the major active components of Herba Cistanche. Previously, Hu et al (17) indicated that $\mathrm{CPhGs}$ could attenuate liver injury in $\mathrm{H} 22$ tumor-bearing mice and inhibit the growth of cancer cells. It was suggested that the underlying mechanism may be related to a reduction in serum $\alpha$-fetoprotein and could enhance immunity in the mice.

In the present study, a hypoxic model of HepG2 liver cancer cells was induced using $\mathrm{CoCl}_{2}$. On this basis, the present study aimed to investigate the effects of OXA on the proliferation, apoptosis, migration and invasion of cancer cells in the presence of CPhGs under hypoxic conditions. In addition, the mRNA and protein expression levels of HIF- $1 \alpha$, LOXL2, E-cadherin and Twist were detected. Moreover, the exact mechanisms underlying the effects of $\mathrm{CPhGs}$ on the pathogenesis of liver cancer were investigated.

\section{Materials and methods}

Cell line. The liver cancer cell line HepG2, as identified using the STR method, was provided by the Clinical Research Institution, First Affiliated Hospital of Xinjiang Medical University (Urumqi, China). The cells were cultured in high-glucose DMEM (HyClone; Cytiva) containing 10\% fetal bovine serum (FBS; Hyclone; Cytiva) at $37^{\circ} \mathrm{C}$ in an incubator containing $5 \% \mathrm{CO}_{2}$.

Preparation of CPhGs. C. tubulosa extraction (CPhGs) was obtained from Hetian Dichen Biotech Co., Ltd.. The content of CPhGs was $>80 \%$, among which the content of echinacoside and verbascose was 44.5 and $16.1 \%$, respectively. Stems of C. tubulosa (Schrenk) Wight were collected in October 2016 from Xinjiang, China. The plant was identified by Dr. Junping Hu. All these voucher specimens (no. 201610) have been deposited at the Plant Herbarium, School of Pharmacy, Xinjiang Medical University, Xinjiang, China.

Experimental design. HepG2 cells $\left(5 \times 10^{4} / \mathrm{ml}\right)$ were treated with various concentrations of $\mathrm{CPhGs}(5,25,50,100,200$ and $500 \mu \mathrm{g} / \mathrm{ml})$ for $48 \mathrm{~h}$ at $37^{\circ} \mathrm{C}(24 \mathrm{~h}$ after seeding) to screening CPhGs-L/M/H dose. HepG2 cells $\left(5 \times 10^{4} / \mathrm{ml}\right)$ were divided into the following groups: i) Control group, cultured in high-glucose DMEM; ii) DMSO group, cultured in $0.1 \%$ DMSO (v/v); iii) $\mathrm{CoCl}_{2}$ group (hypoxia model group), cultured in serum-free DMEM containing $100 \mu \mathrm{M} \mathrm{CoCl}_{2}$; iv) OXA group (positive control group), cultured in $5 \mu \mathrm{M}$ OXA (Beijing Solarbio Science \& Technology Co., Ltd.); v) $\mathrm{OXA}+\mathrm{CoCl}_{2}$ group, cultured in $5 \mu \mathrm{M}$ OXA combined with $100 \mu \mathrm{M} \mathrm{CoCl}_{2}$; and vi) $\mathrm{CPhGs}$ groups, treated with $\mathrm{CPhGs-L/M} / \mathrm{H}(25,50$ and $100 \mu \mathrm{g} / \mathrm{ml}$, respectively) combined with $5 \mu \mathrm{M}$ OXA and $100 \mu \mathrm{M} \mathrm{CoCl}_{2}$.

Cell viability assay. Cell viability was determined using a Cell Counting Kit-8 (CCK-8) assay according to the manufacturer's instructions (Beijing Solarbio Science \& Technology Co., Ltd.) and as previously described (18). Cells $\left(2.0 \times 10^{3}\right.$ cells/well) were seeded in 96 -well plates. Subsequently, $24 \mathrm{~h}$ after seeding, cells were treated for $48 \mathrm{~h}$ according to the treatment conditions in each group. The medium was then replaced with $100 \mu \mathrm{l}$ high-glucose DMEM, followed by the addition of $10 \mu \mathrm{l}$ CCK- 8 reagent; the cells were incubated for $1 \mathrm{~h}$ at $37^{\circ} \mathrm{C}$. Optical density was measured using a multi-detection microplate reader (Thermo Fisher Scientific, Inc.) at $450 \mathrm{~nm}$. Six replicates were prepared for each condition.

Determination of apoptotic rate. Apoptotic rate was determined using an Annexin V/PI Apoptotic Detection Kit (Beijing Solarbio Science \& Technology Co., Ltd.). HepG2 cells $\left(5 \times 10^{5}\right)$ were inoculated in 6-well plates at $37^{\circ} \mathrm{C}$ in $5 \% \mathrm{CO}_{2}$. Subsequently, $24 \mathrm{~h}$ after treatment, the cells were cultured in serum-free DMEM for $4 \mathrm{~h}$. The cells were then digested using $0.25 \%$ trypsinized (Gibco; Thermo Fisher Scientific, Inc.), followed by at least three washes with pre-cooled PBS. Upon centrifugation at $167.7 \mathrm{x} \mathrm{g}$ for $5 \mathrm{~min}$ at $4^{\circ} \mathrm{C}$, the cells were resuspended with $1 \mathrm{X}$ binding buffer and the concentration was adjusted to $1 \sim 5 \times 10^{6} / \mathrm{ml}$, and then stained with $5 \mu 1$ Annexin V-FITC and $5 \mu \mathrm{l}$ PI for $15 \mathrm{~min}$ at room temperature. The cells then underwent flow cytometry using a BD LSRFortessa flow cytometer (BD Biosciences) and FlowJo 10.6.2 software (Tree Star, Inc.).

Wound-healing assay. The inhibitory effects of CPhGs on cell migration were examined by wound-healing assay (19). The cells were seeded into 6-well plates until a 100\% confluent monolayer was obtained. Subsequently, the cells were wounded using a 200- $\mu$ l pipette tip, washed with PBS and incubated with treatments in serum-free medium. After drug treatment, the wound-healing speed was measured at $0,12,24$ and $48 \mathrm{~h}$, respectively. Wound images were obtained using a fluorescent microscope (Nikon Ti-S, Japan) under a magnification of x10. Wound closure was measured by the wound distance in each period and expressed as percentage of the initial wound distance at $0 \mathrm{~h}$.

Transwell assays. Cell invasion was assessed by Transwell assays. Cells $\left(1 \times 10^{5}\right.$ cells $\left./ \mathrm{ml}\right)$ were suspended in $200 \mu \mathrm{l}$ high-glucose DMEM without FBS. The cells were then seeded onto Matrigel-coated upper wells covered with a polyethylene terephthalate filter membrane (pore size, $8.0 \mu \mathrm{m}$ ). A total of $500 \mu \mathrm{l}$ high-glucose DMEM containing 10\% FBS was placed in the lower chamber. Cotton swabs were used to remove the cells on the upper surface of the filter after $48 \mathrm{~h}$ at $37^{\circ} \mathrm{C}$. The cells that invaded through the membrane were fixed with $4 \%$ paraformaldehyde for $30 \mathrm{~min}$. Subsequently, cells were stained with $0.1 \%$ crystal violet for $15 \mathrm{~min}$ at room temperature. Invading cells were observed under a fluorescent microscope (Nikon Ti-S) at a magnification of $\mathrm{x} 100$. 
Table I. Primer sequences.

\begin{tabular}{ll}
\hline Gene & \multicolumn{1}{c}{ Sequence (5'-3') } \\
\hline$\beta$-actin & F: TGGCACCCAGCACAATGAA \\
& R: CTAAGTCATAGTCCGCCTAGAAGCA \\
HIF-1 $\alpha$ & F: CAAGAAACCACCCATGAC \\
& R: GGCTCATAACCCATCAAC \\
LOXL2 & F: ACAGAATGTGAAGGAGACATCC \\
& R: TGATGTTGTGGAGTAATCGGA \\
E-cadherin & F: GACAGGCTGGCTGAAAGTG \\
& R: TGGCTGACGATGGTGTAGG \\
Twist1 & F: GTACATCGACTTCCTCTACCAG \\
& R: CATCCTCCAGACCGAGAAG
\end{tabular}

F, forward; HIF-1 $\alpha$, hypoxia-inducible factor $1 \alpha$; LOXL2, lysyl oxidase-like 2 ; $\mathrm{R}$, reverse.

Reverse transcription-quantitative PCR (RT-qPCR). Total RNA was extracted from HepG2 cells using TRIzol ${ }^{\circledR}$ reagent (Invitrogen; Thermo Fisher Scientific, Inc.) and cDNA synthesis was carried out using the PrimeScript RT reagent kit (Takara Bio, Inc.) according to manufacturer's protocol. qPCR was performed on a 7500 Real-Time PCR system (Applied Biosystems; Thermo Fisher Scientific, Inc.) using TB green $^{\mathrm{TM}}$ Premix Ex Taq ${ }^{\mathrm{TM}}$ (Takara Bio, Inc.) according to manufacturer's protocol. The primers used for qPCR are listed in Table I. PCR conditions consisted of denaturation at $95^{\circ} \mathrm{C}$ for $30 \mathrm{sec}$, followed by 40 cycles of denaturation at $95^{\circ} \mathrm{C}$ for $5 \mathrm{sec}$ and annealing at $60^{\circ} \mathrm{C}$ for $30 \mathrm{sec}$. Finally, the amplification results were analyzed using the $2^{-\Delta \Delta C q}$ method (20).

Western blot analysis. Proteins were extracted from cells treated for $48 \mathrm{~h}$ by homogenization in RIPA lysis buffer (Thermo Fisher Scientific, Inc.) containing protease and phosphatase inhibitors. Cell protein content was determined using the BCA method. Proteins $(40 \mu \mathrm{g})$ were then separated by SDS-PAGE on a $10 \%$ gel and transferred to a PVDF membrane. The membrane was blocked in $5 \%$ nonfat milk for $1 \mathrm{~h}$ at $4^{\circ} \mathrm{C}$, and incubated with the following primary antibodies: $\beta$-actin (1:5,000; cat. no. bs-0061R; BIOSS), HIF-1 $\alpha$ (1:1,000; cat. no. ab179483; Abcam), LOXL2 (1:500; cat. no. ab179810; Abcam), E-cadherin (1:1,000; cat. no. bs-10009R; BIOSS) and Twist1 (1:500; cat. no. bs-2441R; BIOSS) overnight at $4^{\circ} \mathrm{C}$. The membrane was then incubated with goat anti-rabbit IgG H\&L secondary antibodies (1:2,000; cat. no. ab205718; Abcam) for $4 \mathrm{~h}$ at room temperature. After washing with TBS-0.05\% Tween-20, the blots were visualized using the Enhanced Chemiluminescence system (Amersham; Cytiva). The relative intensity of the bands was semi-quantified by densitometric analysis using ImageJ2x software (version 2.1.4.7; Rawak Software Inc.), and densitometric plots of the results were normalized to the intensity of $\beta$-actin.

Statistical analysis. SPSS 19.0 software (SPSS, Inc.) was utilized for data analysis. Data are presented as the mean \pm standard deviation and were analyzed by one-way ANOVA followed by Tukey's post hoc test. $\mathrm{P}<0.05$ was

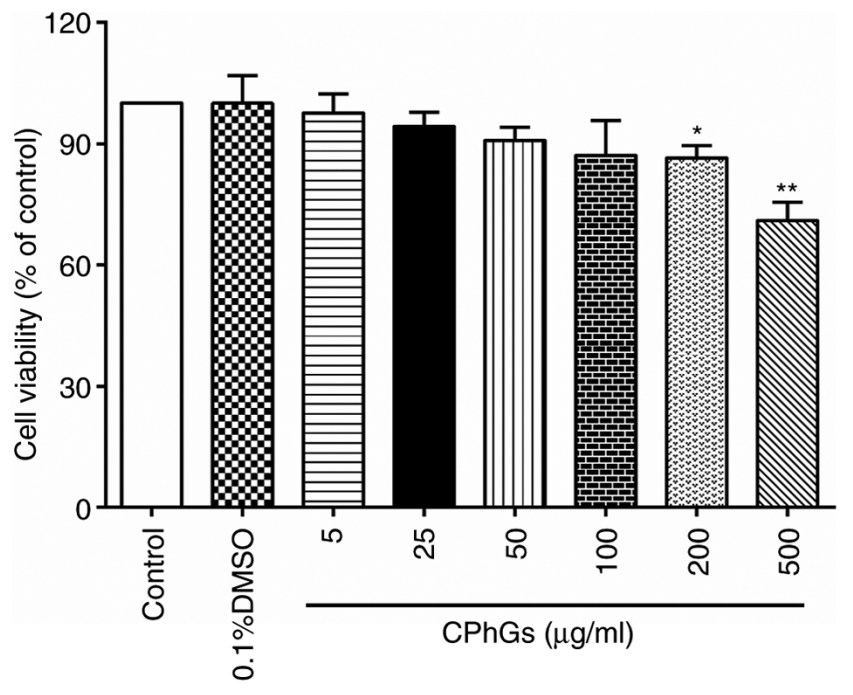

Figure 1. Effects of different concentrations of $\mathrm{CPhGs}$ on HepG2 cell viability after $48 \mathrm{~h} .{ }^{*} \mathrm{P}<0.05,{ }^{* *} \mathrm{P}<0.01$ vs. control group. $\mathrm{CPhGs}$, phenylethanol glycosides from Herba Cistanche.

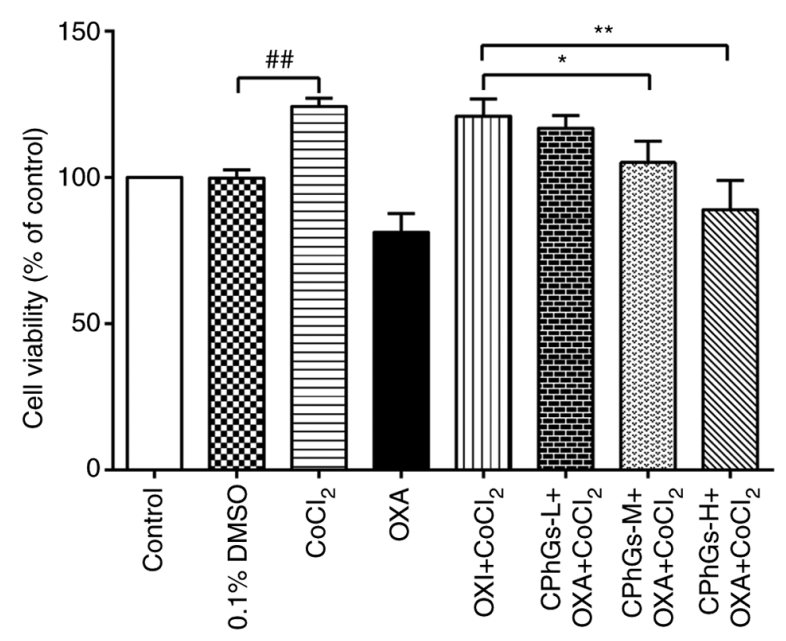

Figure 2. Effects of $\mathrm{CPhGs}$ combined with OXA on the viability of HepG2 cells at 48 h. ${ }^{*} \mathrm{P}<0.05,{ }^{* *} \mathrm{P}<0.01 ;{ }^{\# \#} \mathrm{P}<0.01$. CPhGs, phenylethanol glycosides from Herba Cistanche; H, high; L, low; M, moderate; OXA, oxaliplatin.

considered to indicate a statistically significant difference. All experiments were performed at least in triplicate.

\section{Results}

Effects of CPhGs on cell viability. HepG2 cells were treated with various concentrations of CPhGs $(5,25,50,100,200$ and $500 \mu \mathrm{g} / \mathrm{ml})$ for $48 \mathrm{~h}$ ( $24 \mathrm{~h}$ after seeding). As shown in Fig. 1, there was significant decline in the viability of cells treated with 200 and $500 \mu \mathrm{g} / \mathrm{ml} \mathrm{CPhGs} \mathrm{compared} \mathrm{with} \mathrm{that} \mathrm{of} \mathrm{the}$ control group $(\mathrm{P}<0.05)$. These findings indicated that $\mathrm{CPhGs}$ could modulate cell viability in a dose-dependent manner.

CPhGs enhances the effects of OXA on liver cancer. The effects of $\mathrm{CPhGs}$ on OXA-modulated HepG2 cell viability were subsequently assessed (Fig. 2). After $\sim 48$ h, the combination of CPhGs and OXA significantly decreased the viability of HepG2 cells compared with that in the OXA + 


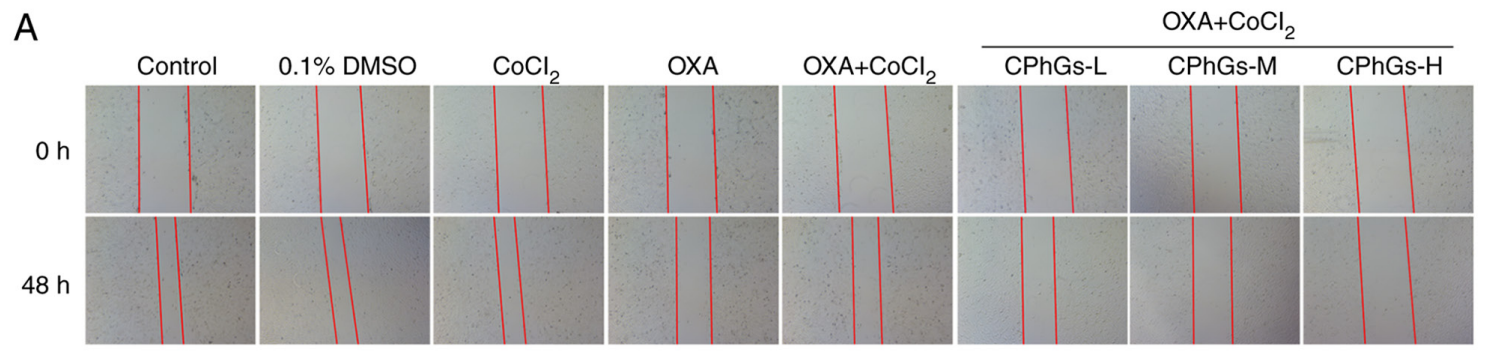

B

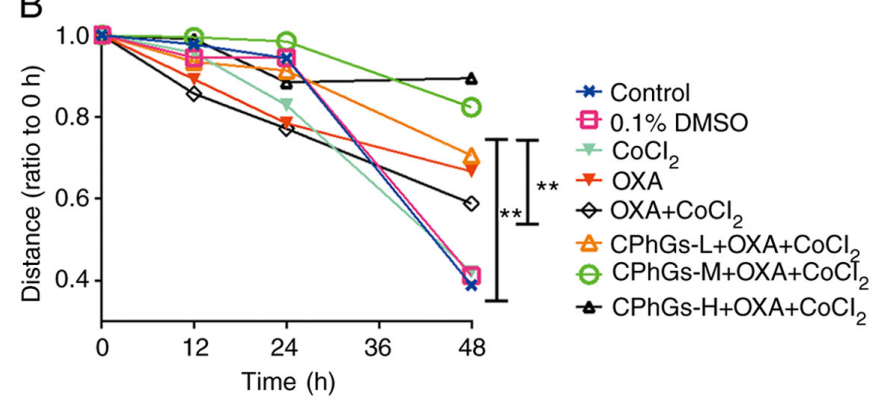

C

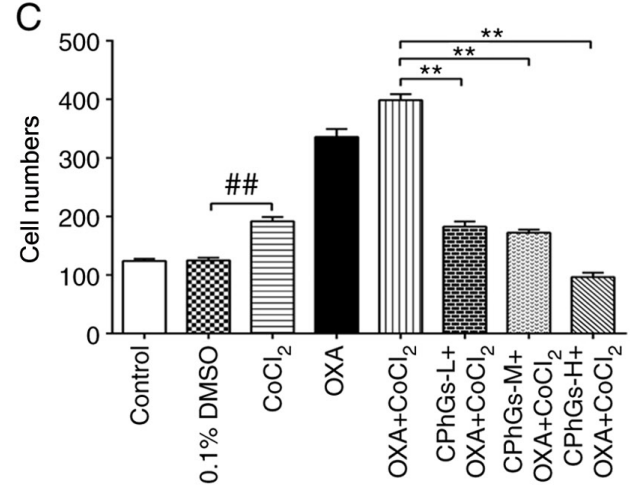

D

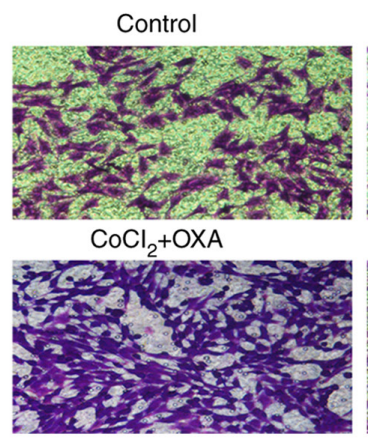

$0.1 \%$ DMSO

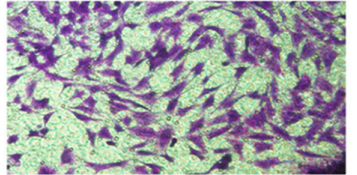

$\mathrm{CPhGs}-\mathrm{L}+\mathrm{OXA}+\mathrm{CoCl}_{2}$

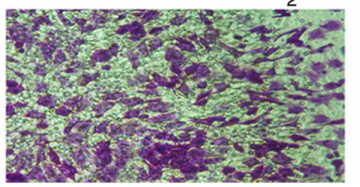

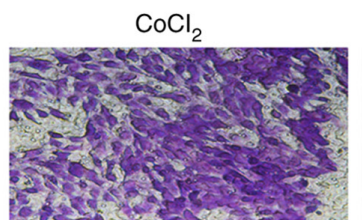

CPhGs-M+OXA+CoCl

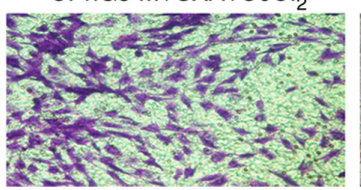

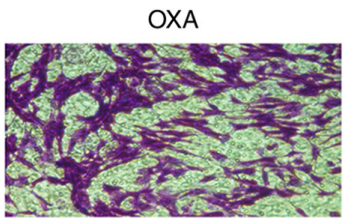

CPhGs-M+OXA+CoCl

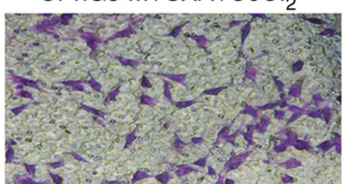

Figure 3. Inhibitory effects of CPhGs combined with OXA on the migration and invasion of HepG2 cells at 48 h. (A and B) Cell migration was measured after treatment with various drugs for $48 \mathrm{~h}$ (magnification, x10). (C) Semi-quantification of cell invasion. (D) Representative images of Transwell cell invasion assays (magnification, x200). ${ }^{* *} \mathrm{P}<0.01 ;{ }^{\# \#} \mathrm{P}<0.01$. CPhGs, phenylethanol glycosides from Herba Cistanche; H, high; L, low; M, moderate; OXA, oxaliplatin.

$\mathrm{CoCl}_{2}$ group. Specifically, CPhGs-M $+\mathrm{OXA}+\mathrm{CoCl}_{2}$ and $\mathrm{CPhGs}-\mathrm{H}+\mathrm{OXA}+\mathrm{CoCl}_{2}$ significantly inhibited the viability of HepG2 cells compared with in the OXA $+\mathrm{CoCl}_{2}$ group $(\mathrm{P}<0.05$ and $\mathrm{P}<0.01$, respectively).

CPhGs inhibit migration and invasion of liver cancer cells. To further study the invasive potential and migratory ability of liver cancer cells following treatment with $\mathrm{CPhGs}$ and OXA, wound-healing and Transwell assays were performed. The wound-healing assay indicated that, compared with that in the DMSO group, the migration of HepG2 cells was reduced following treatment with $\mathrm{CoCl}_{2}$, OXA and $\mathrm{CPhGs}$ (200 or $500 \mu \mathrm{g} / \mathrm{ml})(\mathrm{P}<0.05$; Fig. 3A and B). For the Transwell assay, the invasive ability of cells was markedly inhibited in the co-treatment groups $\left(\mathrm{CPhGs}+\mathrm{OXA}+\mathrm{CoCl}_{2}\right)$ compared with that in the OXA $+\mathrm{CoCl}_{2}$ group $(\mathrm{P}<0.01$; Fig. $3 \mathrm{C}$ and $\mathrm{D})$. Conversely, in the co-treatment groups $\left(\mathrm{CPhGs}+\mathrm{OXA}+\mathrm{CoCl}_{2}\right)$, the invasive potential and migratory ability of cells was markedly inhibited.

Effects of CPhGs and OXA on apoptosis. Following treatment with the combination of $\mathrm{CPhGs}$ and OXA for
$48 \mathrm{~h}$, the HepG2 cells were stained with Annexin V-FITC and PI, followed by flow cytometry to determine cellular apoptosis. As shown in Fig. 4A, the co-treatment groups $\left(\mathrm{CPhGs}-\mathrm{L} / \mathrm{M} / \mathrm{H}+\mathrm{OXA}+\mathrm{CoCl}_{2}\right)$ exhibited a gradual elevation in the proportion of apoptotic cells with the increase in $\mathrm{CPhGs}$ concentration. Most of the cells treated with $\mathrm{CPhGs}$ and OXA were localized in the Q4 region, which indicated that the combination of CPhGs and OXA induced apoptosis at the early stage. Compared with in the $\mathrm{OXA}+\mathrm{CoCl}_{2}$ group, a significant elevation in the apoptotic rate of cells was detected in the groups treated with $\mathrm{OXA}, \mathrm{CoCl}_{2}$ and moderate or high doses of $\mathrm{CPhGs}(\mathrm{P}<0.01$; Fig. 4B). These findings indicated that the combination of OXA and $\mathrm{CPhGs}$ may contribute to the apoptosis of HepG2 cells.

mRNA expression levels of HIF-1 $\alpha$, LOXL2, E-cadherin and Twist following CPhGs and OXA co-incubation. There were no statistical differences in the mRNA expression levels of HIF-1 $\alpha$, LOXL2, E-cadherin and Twist between the control and DMSO groups (P>0.05; Fig. 5A-D). Conversely, $\mathrm{CoCl}_{2}$ induced a significant increase in the mRNA expression levels of LOXL2, HIF-1 $\alpha$ and Twist compared with those in the DMSO 
A
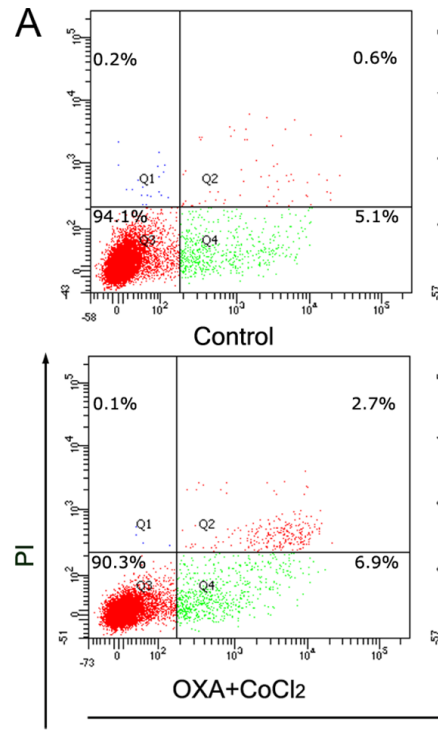

Annexin V-FITC
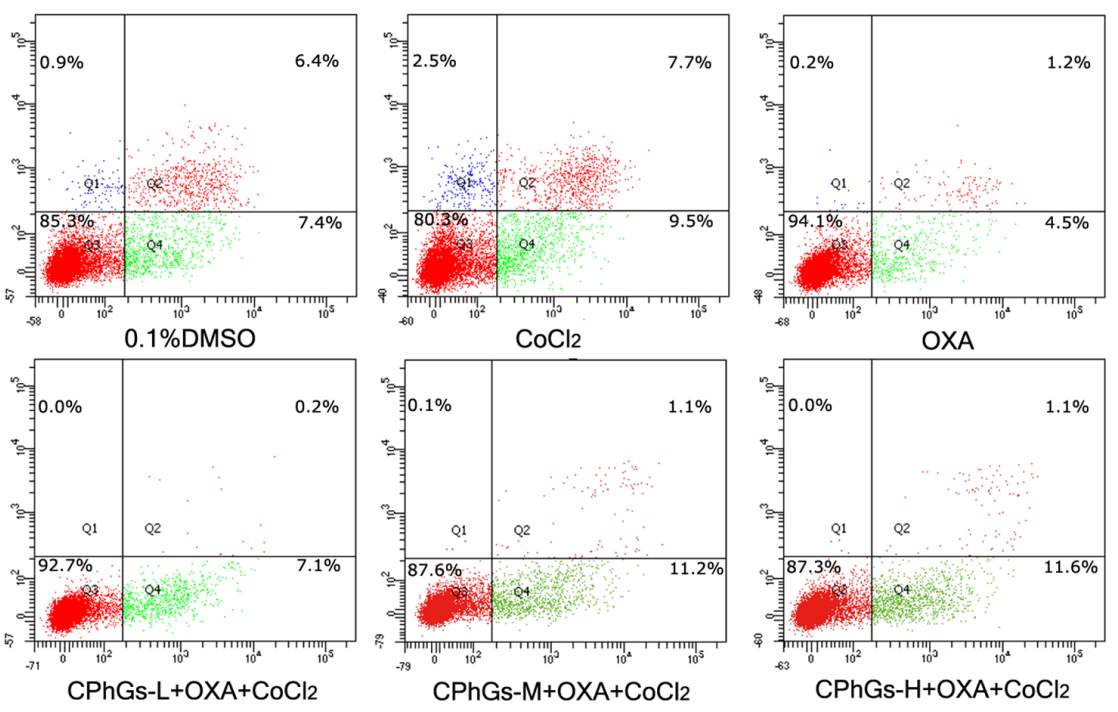

$\mathrm{CPhGs}-\mathrm{H}+\mathrm{OXA}+\mathrm{CoCl}_{2}$

$\mathrm{B}$

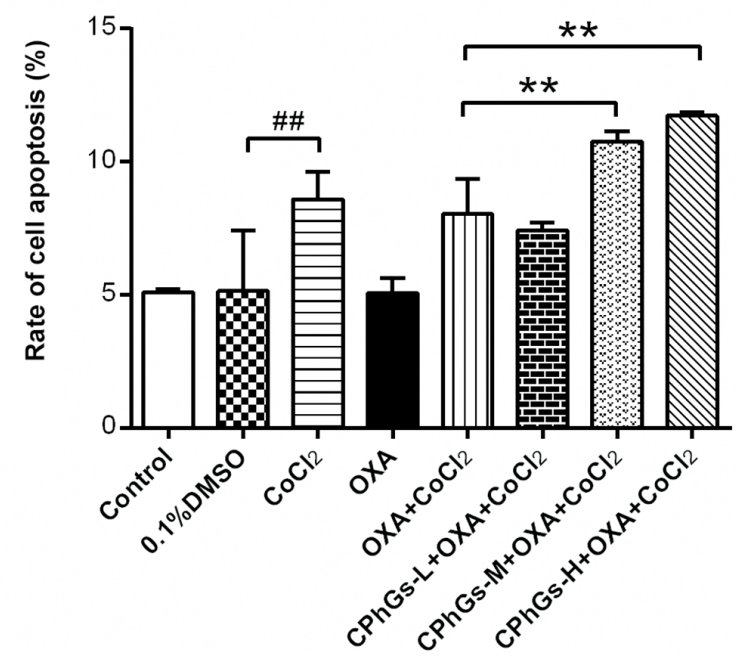

Figure 4. Effect of $\mathrm{CPhGs}$ combined with OXA on the percentage of apoptotic $\mathrm{HepG}_{2}$ cells at $48 \mathrm{~h}$, as determined by Annexin-V/PI double staining. (A) Flow cytometry analysis was performed to determine the effect of CPhGs combined with OXA on cell apoptosis. (B) Quantification of cell apoptotic rates following treatment with CPhGs combined with OXA. Cells were trypsinized and stained with Annexin-V/PI followed by analysis using flow cytometry. Cells in early apoptosis were Annexin-V-positive and PI-negative (Q4). ${ }^{\# \#} \mathrm{P}<0.05 ;{ }^{* *} \mathrm{P}<0.01$. CPhGs, phenylethanol glycosides from Herba Cistanche; H, high; L, low; M, moderate; OXA, oxaliplatin.

group ( $\mathrm{P}<0.01$; Fig. 5A, B and D). Compared with those in the $\mathrm{OXA}+\mathrm{CoCl}_{2}$ group, the mRNA expression levels of LOXL2, HIF-1 $\alpha$ and Twist were significantly enhanced in the CPhGs-H $+\mathrm{OXA}+\mathrm{CoCl}_{2}$ groups $(\mathrm{P}<0.01 ;$ Fig. $5 \mathrm{~A}, \mathrm{~B}$ and $\mathrm{D})$. By contrast, $\mathrm{CoCl}_{2}$ induced a significant downregulation in the mRNA expression levels of E-cadherin compared with those in the DMSO group $(\mathrm{P}<0.01$; Fig. $5 \mathrm{C})$. All concentrations of $\mathrm{CPhGs}$ combined with $\mathrm{OXA}$ and $\mathrm{CoCl}_{2}$ were able to upregulate the mRNA expression levels of E-cadherin compared with those in the $\mathrm{OXA}+\mathrm{CoCl}_{2}$ group $(\mathrm{P}<0.01$; Fig. $5 \mathrm{C})$. These results indicated that the combination of $\mathrm{CPhGs}$ and $\mathrm{OXA}$ effectively inhibited the EMT under hypoxic conditions.

Protein expression levels of HIF-1 $\alpha$, LOXL2, E-cadherin and Twist following CPhGs and OXA co-incubation. The results of western blotting revealed that the protein expression levels of HIF-1 $\alpha$, LOXL2 and Twist were upregulated under hypoxic conditions compared with those in the DMSO group. By contrast, the protein expression levels of E-cadherin were downregulated under hypoxic conditions ( $\mathrm{P}<0.01$; Fig. $6 \mathrm{~A}$ and $\mathrm{B})$. Notably, the protein expression levels of HIF-1 $\alpha$, LOXL2 and Twist were significantly decreased in the CPhGs-M $+\mathrm{OXA}+\mathrm{CoCl}_{2}$ or $\mathrm{CPhGs}-\mathrm{H}+\mathrm{OXA}+\mathrm{CoCl}_{2}$ groups compared with those in the $\mathrm{OXA}+\mathrm{CoCl}_{2}$ group $(\mathrm{P}<0.01$; Fig. $6 \mathrm{~A}$ and $\mathrm{B})$. Compared with DMSO group, $\mathrm{CoCl}_{2}$ treatment significantly decreased the expression level of $\mathrm{E}$-cadherin. In the $\mathrm{CPhGs}$ groups, the protein expression levels of E-cadherin were significantly increased compared with those in the OXA $+\mathrm{CoCl}_{2}$ group $(\mathrm{P}<0.01$; Fig. 6B). These findings indicated that $\mathrm{CPhGs}$ treatment could effectively inhibit the downregulation of E-cadherin, and the upregulation of HIF-1 $\alpha$, LOXL2 and Twist induced by $\mathrm{CoCl}_{2}$.

\section{Discussion}

Hypoxia is a common feature in the cancer microenvironment; this is mainly associated with the fact that proliferation of 
A

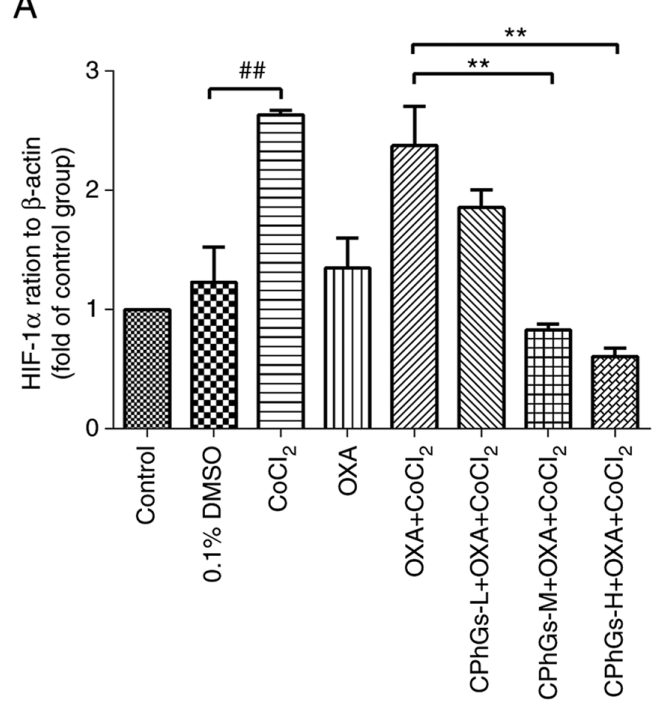

C

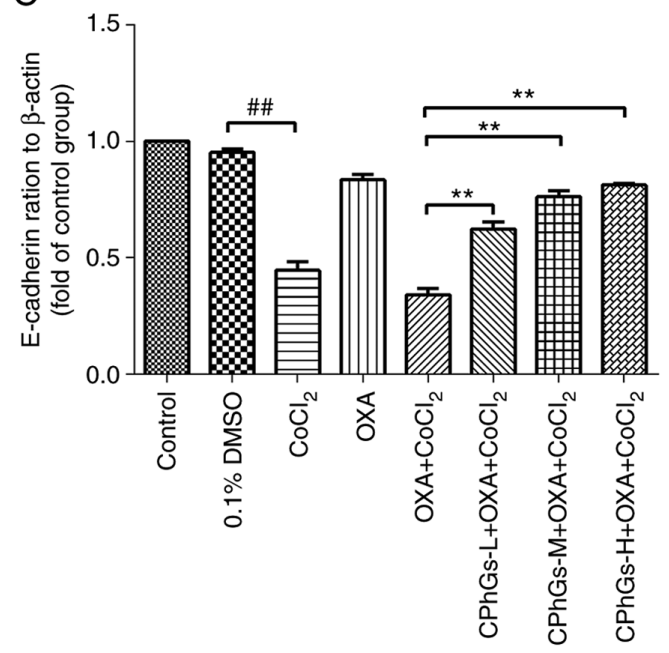

B

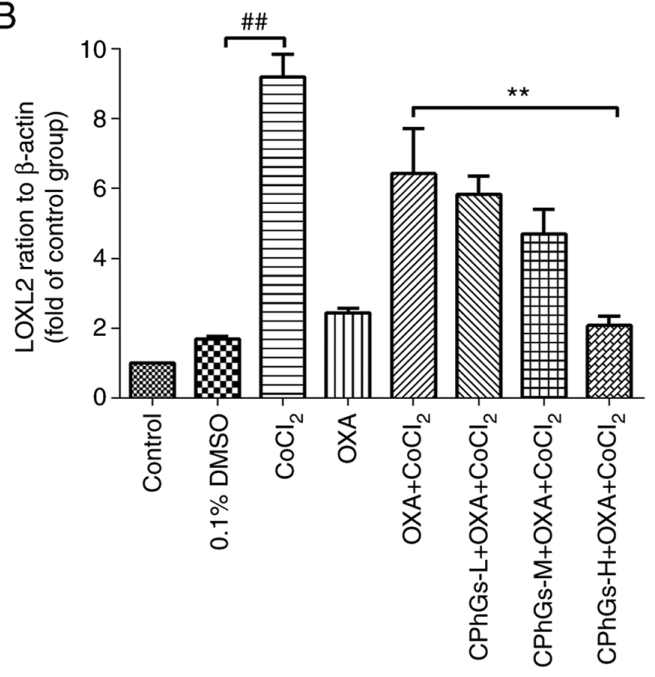

D

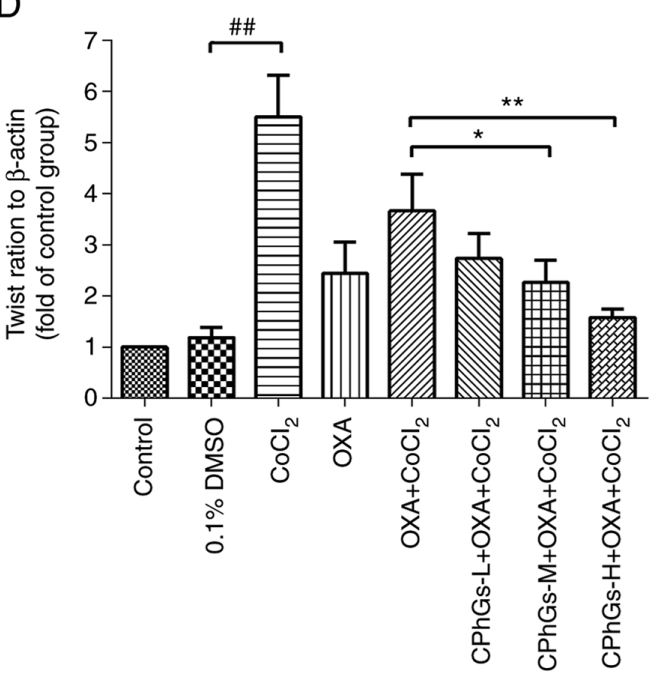

Figure 5. Effect of CPhGs combined with OXA on the mRNA expression levels of HIF-1 $\alpha$, LOXL2, E-cadherin and Twist in HepG2 cells. Effect of CPhGs combined with OXA on (A) HIF-1 $\alpha$, (B) LOXL2, (C) E-cadherin and (D) Twist gene expression in HepG2 cells. ${ }^{\# \#} \mathrm{P}<0.01$; ${ }^{*} \mathrm{P}<0.05,{ }^{* *} \mathrm{P}<0.05$. CPhGs, phenylethanol glycosides from Herba Cistanche; H, high; HIF-1 $\alpha$, hypoxia-inducible factor 1 $\alpha$; L, low; LOXL2, lysyl oxidase-like 2; M, moderate; OXA, oxaliplatin.

cancer cells is more rapid compared with vascular formation of aberrant neovessels. In addition, other biological processes, including proliferation, metastasis and drug sensitivity are affected by hypoxia (21). The tumor hypoxic microenvironment is crucial for the pathogenesis and progression of cancer, and it is also important in the drug resistance and vascularization of liver cancer (22).

In the present study, HepG2 cells were treated with various concentrations of CPhGs, among which CPhGs $(200 \mu \mathrm{g} / \mathrm{ml})$ could significantly induce a decrease in cell viability. Notably, $\mathrm{CPhGs}$ could modulate cellular viability in a dose-dependent manner. Under hypoxic conditions, the combination of OXA and CPhGs (50 or $100 \mu \mathrm{g} / \mathrm{ml})$ significantly inhibited the viability of HepG2 cells compared with OXA treatment alone. A similar dose-dependent trend was observed in the migration and invasion assays of liver cancer cells. Currently, extensive studies have been conducted to investigate the roles of cancer cell apoptosis in the pathogenesis of liver disease (23-26). Several strategies have been developed for treating liver cancer by promoting apoptosis (27-29); therefore, interference in
HepG2 cell apoptosis may serve as a promising candidate for the prevention and treatment of liver cancer. The apoptosis of HepG2 cells was significantly enhanced following treatment with the combination of CPhGs-M/-H and OXA compared with that in cells treated with OXA alone. Therefore, it was indicated that $\mathrm{CPhGs}$ could significantly enhance the antitumor affects of OXA.

HIF-1 $\alpha$ can upregulate the expression levels of E-cadherin, $\mathrm{N}$-cadherin and Vimentin, as well as some transcription factors, such as Snail1/2, Zeb1 and Twist1. Subsequently, this might lead to a loss of cellular polarity, loosening of cell-cell junctions, alterations in cytoskeletal protein, and the migration and invasion of cancer cells, which could result in the translocation of cancer cells to the circulatory system through the basilar membrane and subsequent metastasis (30). In the present study, $\mathrm{CoCl}_{2}$ was used to induce a model of hypoxia, which triggered an increase in the viability of HepG2 cells, as well as cell migration and invasion. Furthermore, the mRNA and protein expression levels of HIF-1 $\alpha$ were significantly increased, indicating that $\mathrm{CoCl}_{2}$ induced the generation of a 
A
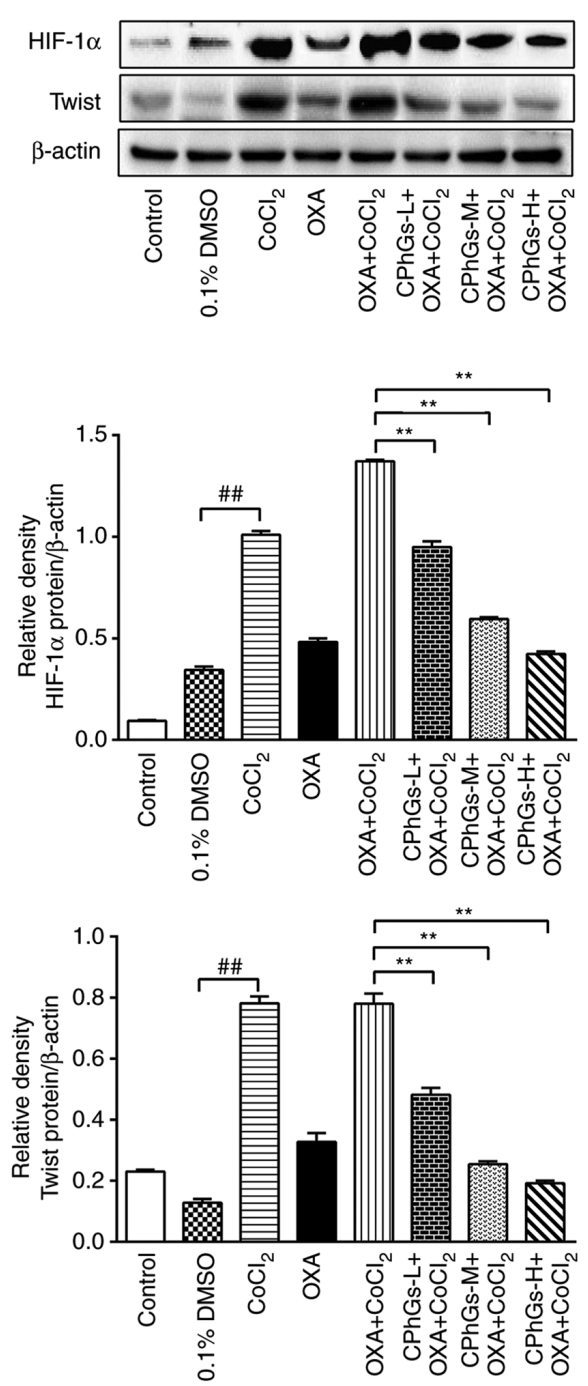

B
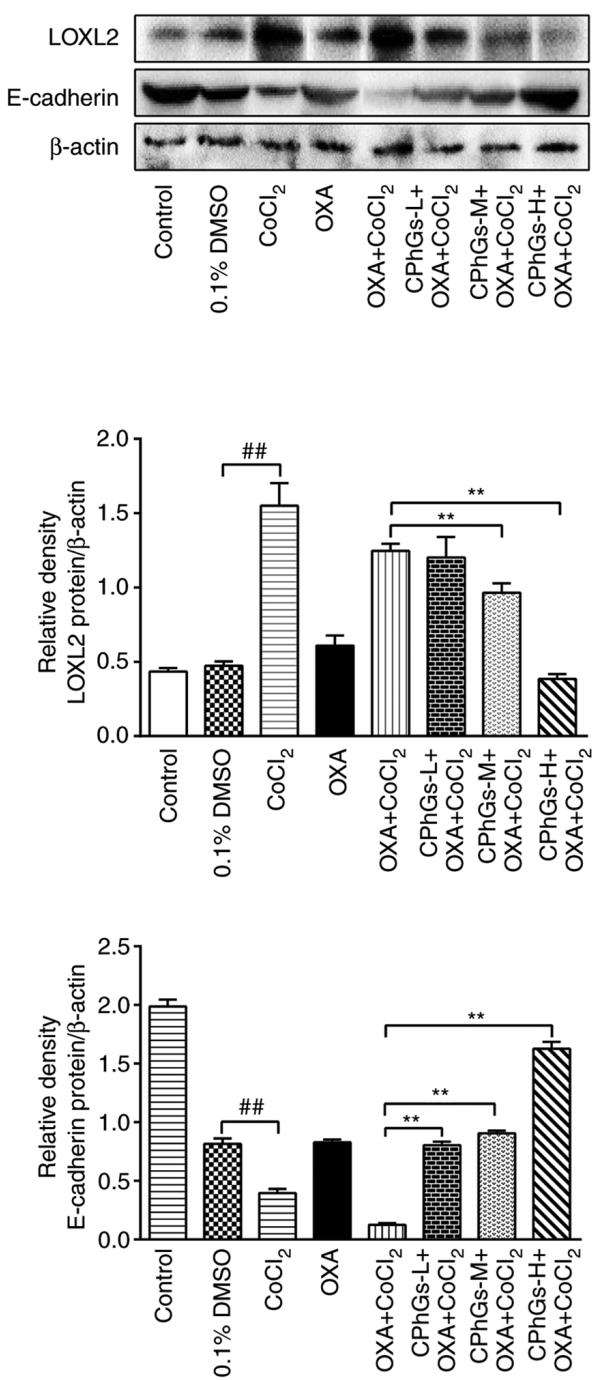

Figure 6. Protein expression levels of HIF-1 $\alpha$, LOXL2 and EMT-associated biomarkers (E-cadherin and Twist) after CPhGs and OXA co-incubation. (A) Protein expression levels of HIF-1 $\alpha$ and Twist in HepG2 cells following CPhGs and OXA co-incubation. (B) Protein expression levels of LOXL2 and E-cadherin in HepG2 cells following CPhGs and OXA co-incubation. ${ }^{\# \#} \mathrm{P}<0.01 ;{ }^{* *} \mathrm{P}<0.01$. CPhGs, phenylethanol glycosides from Herba Cistanche; H, high; HIF-1 $\alpha$, hypoxia-inducible factor $1 \alpha$; L, low; LOXL2, lysyl oxidase-like 2; M, moderate; OXA, oxaliplatin.

hypoxic microenvironment. Treatment with the combination of CPhGs and OXA markedly inhibited the mRNA and protein expression levels of HIF-1 $\alpha$ induced by hypoxia. These findings suggested that $\mathrm{CPhGs}$ could attenuate the microenvironment of liver cancer in a dose-dependent manner.

E-cadherin is a $\mathrm{Ca}^{2+}$-dependent adhesion molecule, which has a key role in cell-cell adhesion, maintenance of integrity of tissue structure and signaling transmission. In cases of downregulation or even loss of adhesion function, cancer cells may exhibit uncontrolled proliferation and dedifferentiation, which may promote increased invasion of cancer cells and subsequent metastasis (31). In addition, E-cadherin is crucial for inhibiting the EMT of cancer cells, which is closely associated with the differentiation, invasion, metastasis and prognosis of multiple epithelial malignancies. EMT-inducing transcription factors (EMT-TFs), such as Twist, Snail and Zeb, are crucial for EMT. Hypoxia has been reported to activate signaling pathways that induce EMT-TF expression; notably, it could directly promote EMT via the transcriptional activation of these factors (32). Twist is a highly conserved helix-ring-helix transcription factor that has been newly identified in recent years. High Twist expression has been detected in numerous types of cancer cells (33). Therefore, it is essential to investigate the association between Twist expression and the migration or metastasis of cancer cells, as well as clinical prevention and treatment of metastasis (34). In the present study, it was revealed that the mRNA and protein expression levels of E-cadherin were downregulated in the presence of hypoxia, whereas the mRNA and protein expression levels of Twist were elevated. These findings were consistent with the results of invasion and migration assays, which implied that hypoxia may contribute to the occurrence of EMT. Following treatment with OXA, the protein expression levels of E-cadherin were downregulated; this indicated that OXA exhibited poor efficiency in inhibiting the growth of liver cancer cells, whereas it could promote the EMT. However, in combination with $\mathrm{CPhGs}$, the sensitivity of HepG2 cells to OXA exhibited marked improvement in the presence of hypoxia. Furthermore, cotreatment with CPhGs and OXA could inhibit cellular viability, migration and invasion of HepG2 cells. The present study only detected 
Twist and E-cadherin expression; therefore, future studies aim to focus on more EMT-related markers, in order to evaluate the inhibitory effect of CPhGs on hypoxia-induced EMT in liver cancer.

HIF-1 $\alpha$ has been reported to promote the expression of LOXL2, and to enhance the migration and invasion of hepatic cancer cells, which may be closely related to the poor prognosis of liver cancer (30). In a previous study, the expression levels of LOXL2 in adjacent liver cancer tissues were markedly increased compared with those in the cancer tissues (35). In addition, it was closely related to the invasion and metastasis of liver cancer. LOXL2 gene silencing by small interfering RNA inhibited the proliferation of HepG2 and SMCC-7721 cells, which resulted in cell cycle arrest of cancer cells and increased apoptosis (36). Shao et al (35) investigated the correlation between LOXL2 in liver cancer samples, and clinicopathological factors, VM and prognosis among 201 cases that received surgery for treatment. It was hypothesized that LOXL2 served important roles in the pathogenesis and progression of liver cancer, which may serve as a target for drug development. Furthermore, Peng et al (37) demonstrated that LOXL2 could activate the Snail/E-cadherin and Src kinase/Focal adhesion kinase signaling pathways, which may contribute to the pathogenesis and progression of EMT of gastric cancer cells. In the present study, under hypoxic conditions, the mRNA and protein expression levels of LOXL2 were increased, whereas its expression was downregulated following treatment with OXA. Furthermore, treatment with a combination of $\mathrm{CPhGs}$ and $\mathrm{OXA}$ resulted in obvious downregulation of LOXL2 expression, which may effectively aid the antitumor effects of OXA on liver cancer.

There are some limitations to the present study. The present study should have used two more liver cancer cell lines, including the SMCC-7721 cell line, but these could not be used as it was not possible to purchase these cell lines because they were misidentified and were derived from HeLa cells. In addition, the present study did not analyze the antioxidant effects of different concentrations of CPhGs in the cells.

In conclusion, $\mathrm{CPhGs}$ could alternate the hypoxic tumor microenvironment of liver cancer cells through modulating the HIF-1 $\alpha$ signaling pathway. In addition, the sensitivity of liver cancer cells to OXA was significantly elevated in response to treatment with a combination of $\mathrm{CPhGs}$ and OXA. These findings may provide a novel treatment strategy to improve the sensitivity of liver cancer to chemotherapy.

\section{Acknowledgements}

Not applicable.

\section{Funding}

The present study was supported by the Xinjiang Key Laboratory of Natural Drug Active Components and Drug Release Technology (grant no. XJDX1713), the Reserve Candidate Project for the Leader of Scientific and Technological Innovation in Xinjiang Uygur Autonomous Region (grant no. 2019XS14), the National Natural Science Foundation of China (grant no. 81860735) and the Bethune Charitable
Foundation 'Bethune-Quest-construction of pharmaceutical scientific research capacity' (grant no. B-19-H-20200622).

\section{Availability of data and materials}

The datasets used and/or analyzed during the current study are available from the corresponding author on reasonable request.

\section{Authors' contributions}

LMW and JWZ performed the experiments, drafted the manuscript and confirmed the authenticity of all the raw data. JPH and JHY designed the present study. All authors read and approved the final manuscript.

\section{Ethics approval and consent to participate}

Not applicable.

\section{Patient consent for publication}

Not applicable.

\section{Competing interests}

The authors declare that they have no competing interests.

\section{References}

1. Hepatocellular carcinoma. Nat Rev Dis Primers 2: 16019, 2016.

2. Gingold JA, Zhu D, Lee DF, Kaseb A and Chen J: Genomic profiling and metabolic homeostasis in primary liver cancers. Trends Mol Med 24: 395-411, 2018.

3. McGuire S: World cancer report 2014. Geneva, Switzerland: World health organization, international agency for research on cancer, WHO press, 2015. Adv Nutr 7: 418-419, 2016.

4. Gholamreza K, Jadidi-Niaragh F, Jahromi AS, Zandi K and Hojjat-Farsangi M: Mechanisms of tumor cell resistance to the current targeted-therapy agents. Tumour Biol 37: 10021-10039, 2016.

5. Dong $X$ and Mumper RJ: Nanomedicinal strategies to treat multidrug-resistant tumors: Current progress. Nanomedicine (Lond) 5: 597-615, 2010.

6. Xie Y and Zhong DW: AEG-1 is associated with hypoxia-induced hepatocellular carcinoma chemoresistance via regulating PI3K/AKT/HIF-1alpha/MDR-1 pathway. EXCLI J 15: 745-757, 2016.

7. Xiong H, Ni Z, He J, Jiang S, Li X, He J, Gong W, Zheng L, Chen $\mathrm{S}, \mathrm{Li} \mathrm{B}$, et al: LncRNA HULC triggers autophagy via stabilizing Sirt1 and attenuates the chemosensitivity of HCC cells. Oncogene 36: 3528-3540, 2017.

8. Gade TPF, Tucker E, Nakazawa MS, Hunt SJ, Wong W, Krock B Weber CN, Nadolski GJ, Clark TWI, Soulen MC, et al: Ischemia induces quiescence and autophagy dependence in hepatocellular carcinoma. Radiology 283: 702-710, 2017.

9. Siegel RL, Miller KD and Jemal A: Cancer statistics, 2017. CA Cancer J Clin 67: 7-30, 2017.

10. Dong LQ, Shen BQ and Ma Y: Research progress of hypoxia microenvironment in hepatocellular carcinoma. Zhong Guo Pu Wai Ji Chu Yu Lin Chuang Za Zhi 25: 1254-1258, 2018 (In Chinese).

11. Moon HJ, Finney J, Ronnebaum T and Mure M: Human lysyl oxidase-like 2. Bioorg Chem 57: 231-241, 2014

12. Ferreira S, Saraiva N, Rijo P and Fernandes AS: LOXL2 inhibitors and breast cancer progression. Antioxidants (Basel) 10: 312,2021

13. Philp CJ, Siebeke I, Clements D, Miller S, Habgood A, John AE, Navaratnam V, Hubbard RB, Jenkins G and Johnson SR: Extracellular matrix cross-linking enhances fibroblast growth and protects against matrix proteolysis in lung fibrosis. Am J Respir Cell Mol Biol 58: 594-603, 2018. 
14. Galván JA, Zlobec I, Wartenberg M, Lugli A, Gloor B, Perren A and Karamitopoulou E: Expression of E-cadherin repressors SNAIL, ZEB1 and ZEB2 by tumour and stromal cells influences tumour-budding phenotype and suggests heterogeneity of stromal cells in pancreatic cancer. Br J Cancer 112: 1944-1950, 2015.

15. Gu C, Yang X and Huang L: Cistanches herba: A neuropharmacology review. Front Pharmacol 7: 289, 2016

16. Fu Z, Fan X, Wang X and Gao X: Cistanches Herba: An overview of its chemistry, pharmacology, and pharmacokinetics property. J Ethnopharmacol 219: 233-247, 2018.

17. Hu Q, You SP, Liu T, Wang B, Liu X and Jiang Y: An investigation on the anti-liver cancer effect of cistanche. Carcinog Teratog Mutagen 30: 194-199, 2018.

18. Mao J, Tian Y, Wang C, Jiang K, Li R, Yao Y, Zhang R, Sun D, Liang $\mathrm{R}, \mathrm{Gao} Z$, et al: $\mathrm{CBX} 2$ regulates proliferation and apoptosis via the phosphorylation of YAP in hepatocellular carcinoma. J Cancer 10: 2706-2719, 2019.

19. Qin Y, Liu HJ, Li M, Zhai DH, Tang YH, Yang L, Qiao KL, Yang JH, Zhong WL, Zhang Q, et al: Salidroside improves the hypoxic tumor microenvironment and reverses the drug resistance of platinum drugs via HIF-1 $\alpha$ signaling pathway. EBioMedicine 38: 25-36, 2018.

20. Livak KJ and Schmittgen TD: Analysis of relative gene expression data using real-time quantitative PCR and the 2(-Delta Delta C(T)) method. Methods 25: 402-408, 2001.

21. Vaupel P: Tumor microenvironmental physiology and its implications for radiation oncology. Semin Radiat Oncol 14: 198-206, 2004

22. Chen C and Lou T: Hypoxia inducible factors in hepatocellular carcinoma. Oncotarget 8: 46691-46703, 2017.

23. Schwabe RF and Luedde T: Apoptosis and necroptosis in the liver: A matter of life and death. Nat Rev Gastroenterol Hepatol 15: 738-752, 2018.

24. Kanda T, Matsuoka S, Yamazaki M, Shibata T, Nirei K, Takahashi H, Kaneko T, Fujisawa M, Higuchi T, Nakamura $\mathrm{H}$, et al: Apoptosis and non-alcoholic fatty liver diseases. World J Gastroenterol 24: 2661-2672, 2018.

25. Pittala S, Krelin Y and Shoshan-Barmatz V: Targeting liver cancer and associated pathologies in mice with a mitochondrial VDAC1-based Peptide. Neoplasia 20: 594-609, 2018.

26. Jing ZT, Liu W, Xue CR, Wu SX, Chen WN, Lin XJ and Lin X AKT activator SC79 protects hepatocytes from TNF- $\alpha$-mediated apoptosis and alleviates d-Gal/LPS-induced liver injury. Am J Physiol Gastrointest Liver Physiol 316: G387-G396, 2019.
27. Zhu YJ, Zheng B, Wang HY and Chen L: New knowledge of the mechanisms of sorafenib resistance in liver cancer. Acta Pharmacol Sin 38: 614-622, 2017.

28. Wei R, Cao J and Yao S: Matrine promotes liver cancer cell apoptosis by inhibiting mitophagy and PINK1/Parkin pathways. Cell Stress Chaperones 23: 1295-1309, 2018.

29. Shen L, Zhang G, Lou Z, Xu G and Zhang G: Cryptotanshinone enhances the effect of arsenic trioxide in treating liver cancer cell by inducing apoptosis through downregulating phosphorylated-STAT3 in vitro and in vivo. BMC Complement Altern Med 17: 106, 2017.

30. Wu F, Zhang J, Liu Y, Zheng Y and Hu N: HIF1 $\alpha$ genetic variants and protein expressions determine the response to platinum based chemotherapy and clinical outcome in patients with advanced NSCLC. Cell Physiol Biochem 32: 1566-1576, 2013.

31. Heerboth S, Housman G, Leary M, Longacre M, Byler S, Lapinska K, Willbanks A and Sarkar S: EMT and tumor metastasis. Clin Transl Med 4: 6, 2015.

32. Hapke RY and Haake SM: Hypoxia-induced epithelial to mesenchymal transition in cancer. Cancer Lett 487: 10-20, 2020.

33. Zhao Z, Rahman MA, Chen ZG and Shin DM: Multiple biological functions of Twist1 in various cancers. Oncotarget 8: 20380-20393, 2017.

34. Goossens S, Vandamme N, Van Vlierberghe P and Berx G: EMT transcription factors in cancer development re-evaluated: Beyond EMT and MET. Biochim Biophys Acta Rev Cancer 1868: 584-591, 2017.

35. Shao B, Zhao X, Liu T, Zhang Y, Sun R, Dong X, Liu F, Zhao N, Zhang $\mathrm{D}, \mathrm{Wu} \mathrm{L}$, et al: LOXL2 promotes vasculogenic mimicry and tumour aggressiveness in hepatocellular carcinoma. J Cell Mol Med 23: 1363-1374, 2019.

36. Wu L, Zhang Y, Zhu Y, Cong Q, Xiang Y and Fu L: The effect of LOXL2 in hepatocellular carcinoma. Mol Med Rep 14: 1923-1932, 2016

37. Peng L, Ran YL, Hu H, Yu L, Liu Q, Zhou Z, Sun YM, Sun LC, Pan J, Sun LX, et al: Secreted LOXL2 is a novel therapeutic target that promotes gastric cancer metastasis via the Src/FAK pathway. Carcinogenesis 30: 1660-1669, 2009.

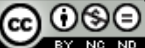

This work is licensed under a Creative Commons Attribution-NonCommercial-NoDerivatives 4.0 International (CC BY-NC-ND 4.0) License. 